\title{
Elevate to Alleviate - Evidence Based Vascular Nursing Study
}

\author{
Nicola lelapi ${ }^{1,2, *}$, Michele Andreucci, ${ }^{3, *}$, Umberto Marcello Bracale ${ }^{4}$, Davide Costa ${ }^{1,5}$, \\ Egidio Bevacqua ${ }^{6}$, Nicola Giannotta ${ }^{1,6}$, Maria Grazia Bevacqua ${ }^{7}$, Giuseppe Filiberto Serraino ${ }^{7}$, \\ Pasquale Mastroroberto ${ }^{7}$, Michele Provenzano ${ }^{6}$, Raffaele Serra (1D) ${ }^{1,6}$ \\ 'Interuniversity Center of Phlebolymphology (CIFL), International Research and Educational Program in Clinical and Experimental Biotechnology" at \\ the Department of Surgical and Medical Sciences, University Magna Graecia of Catanzaro, Catanzaro, Italy; ${ }^{2}$ Department of Public Health and \\ Infectious Disease, Sapienza University of Rome, Rome, Italy; ${ }^{3}$ Department of Health Sciences, University Magna Graecia of Catanzaro, Catanzaro, \\ Italy; ${ }^{4}$ Department of Public Health, Vascular Surgery Unit, University Federico II of Naples, Naples, Italy; ${ }^{5}$ Department of Law, Economics and \\ Sociology, University Magna Graecia of Catanzaro, Catanzaro, Italy; ${ }^{6}$ Department of Medical and Surgical Sciences, University Magna Graecia of \\ Catanzaro, Catanzaro, 88100, Italy; ${ }^{7}$ Department of Experimental and Clinical Medicine, University Magna Graecia of Catanzaro, Catanzaro, Italy
}

*These authors contributed equally to this work

Correspondence: Raffaele Serra, Department of Medical and Surgical Sciences, University Magna Graecia of Catanzaro, Catanzaro, Italy, Email rserra@unicz.it

Background: Venous return in the circulatory system of lower limbs results from the interaction of several mechanisms and reflects the balance between blood inflow and outflow. Blood outflow improves during the lying position especially with leg elevation both in patients with chronic venous disease (CVD) and in healthy subjects in sedentary settings with short-term immobilization. This study evaluated the level of comfort of hospital inpatients, without CVD, lying with a moderate leg elevation during hospital stay.

Methods: This was a clinical trial examining 60 consecutive patients referred to vascular surgery, cardiac surgery, and nephrology units. After inclusion, patients were randomly assigned to two groups: A (leg elevation) in which patients were made to lie in the hospital bed with moderate elevation of the legs, and B (no leg elevation) in which patients were made to lie in the hospital bed without leg elevation.

Results: The whole population consisted of 40 patients undergoing leg elevation (group A) and 20 without leg elevation (group B). During each day of hospitalization, measurements such as ankle and calf circumference, heart rate, blood pressure, and body temperature were collected. Subjective data such as perceived heaviness in the lower limbs, comfort perception and hours of sleep were also collected. In this study, patients of group A recorded a decrease in calf and ankle circumference (delta in ankle and calf circumference (difference between end of study visit and baseline visit) were significantly decreased in group A vs group B ( $<0.001$ for both sides), and patients perceived less leg heaviness and even reported more leg comfort compared to patients of Group B ( $\mathrm{p}<0.001)$ ). Among patients of group A, the bigger advantage in terms of comfort perception was recorded in patients with $15^{\circ}$ leg elevation.

Conclusion: Moderate leg elevation during hospital stay seems to effectively improve leg comfort in hospitalized patients.

Keywords: venous system, lower limb, circulation, leg elevation, hospital stay, vein symptoms, leg discomfort

\section{Introduction}

The circulatory system moves blood through the body, and veins are an important part of this system. While the heart pumps blood in arteries, veins must work against gravity to return blood to the heart by means of the activation of muscular pumps of the lower limbs that represent the peripheral heart of the venous system. The action of the muscular pumps, together with the normal functioning of the venous anti-reflux valves system, ensure an adequate venous return to the heart during leg muscle exercise. Nevertheless, leg muscles are not able to function as good as the heart, and any condition that affects normal walking and limb activities, such as short-term immobilization during hospital stay or any 
conditions that induce limited leg mobility, may impair venous return causing an increase in venous pressure and consequent leg discomfort. ${ }^{1-3}$

Moreover, leg position exerts a direct effect on calf venous return during lying position, as changes in calf blood flow significantly increase as the calf region is elevated above the horizontal level, and this is also related to muscle sympathetic nerve activity supplying calf blood vessels that control several hemodynamic variables. ${ }^{4}$ Leg elevation also enhances microcirculatory flow velocity due to a decrease in venous pressure, with subsequent increase in arteriovenous pressure gradient and capillary flow. ${ }^{5}$ These latter mechanisms are highlighted as the skeletal muscle pump is not always obligatory for sustaining venous return but many other mechanisms are involved. ${ }^{6}$

In patients with chronic venous disease (CVD), one of the most common diseases of the superficial venous system affecting many people of the western world, ${ }^{7}$ simple leg elevation results in a drop in venous pressure relieving the related symptoms. ${ }^{8}$

$\mathrm{Li}$ et $\mathrm{al}^{9}$ showed that elevating the leg of patients who had undergone total knee arthroplasty promoted venous return and reduced leg swelling during hospital stay. When the action of the venous pump is temporarily impaired, as in during hospital stay, it is conceivable that some leg discomfort may occur, and, in this case lying with a moderate leg elevation may help to relieve leg discomfort.

The aim of this study was to evaluate the level of comfort of hospital inpatients, without CVD, lying with a moderate leg elevation during their hospital stay.

\section{Methods}

This was an observational cross-sectional multicenter study examining 60 consecutive patients referred to our Vascular Surgery, Cardiac Surgery, and Renal Units at Magna Graecia University of Catanzaro (Italy) from January 1st to July 1st, 2021. The study was approved by the Institutional Review Board (IRB) of CIFL - Interuniversity Center of Phlebolymphology - at Magna Graecia University of Catanzaro (Id approval number: ER.ALL.2018.12.A) and all patients provided signed informed consent. The present study was designed with the aim to evaluate the level of comfort of hospital inpatients, without CVD, if they lie with a moderate leg elevation during hospital stay. To this purpose, patients were included if they met the following criteria: age $>18$ years, in-patients with a hospital stay of at least seven days, absence of chronic venous disease (CVD), absence of any problem related to normal walking, no previous or actual intervention to the lower limbs for any cause. All participants, in order to be included in the study, had to declare that they did not lead a sedentary lifestyle and that they performed at least 30 minutes of physical activity per day. All patients underwent echo duplex scanning of vascular system of lower limbs to exclude CVD. Patients with vascular or osteoarticular leg problems that affect or prevent leg elevation during rest were excluded from the study. After inclusion, patients were randomly assigned to two groups: A (leg elevation) in which patients were made to lie in the hospital bed with moderate elevation of the legs, and B (no leg elevation) in which patients were made to lie in the hospital bed without leg elevation. At the hospitalization visit, data about comorbidities, blood pressure, body mass index (BMI) and diagnosis were collected. During each day of hospitalization, measurements such as ankle and calf circumference, heart rate, blood pressure, body temperature were collected. Subjective data such as perceived heaviness in the lower limbs, comfort perception and hours of sleep were also collected.

For perceived heaviness in the lower limbs and comfort perception, two scales with 0-10 score were used. For perceived heaviness in the lower limbs 0 was minimum perceived heaviness and 10 was maximum perceived heaviness. For comfort perception 0 was minimum comfort perception and 10 was maximum comfort perception.

Environmental conditions and the clothing that the patients wore during the protocols were evaluated in order to not induce hyperthermia, as it is thought to increase limb blood flow through the activation of thermosensitive mechanisms within the limb vasculature. ${ }^{10}$ In particular, environmental temperature of hospital rooms was set at $20^{\circ} \mathrm{C}$, and patients wore light cotton pajamas during hospital stay.

This work fully complies with CONSORT reporting guidelines: http://www.consort-statement.org, last accessed on 14 October 2021. ${ }^{11}$ 
Table I Baseline Characteristics of Patients According to Type of In-Hospital Stay (Group A Refers to Patients with Leg Elevation and Group B Refers to Patients without Leg Elevation)

\begin{tabular}{|c|c|c|c|}
\hline & $\begin{array}{c}\text { Group A } \\
(\mathrm{N}=40)\end{array}$ & $\begin{array}{c}\text { Group B } \\
(\mathbf{N}=\mathbf{2 0})\end{array}$ & $P$ \\
\hline Age (years) & $62.3 \pm 11.7$ & $63.9 \pm 9.4$ & 0.581 \\
\hline Men (\%) & 80.0 & 70.0 & 0.388 \\
\hline Body Mass Index $\left(\mathrm{kg} / \mathrm{m}^{2}\right)$ & $26.9 \pm 3.0$ & $26.7 \pm 3.4$ & 0.882 \\
\hline Diabetes (\%) & 27.5 & 45.0 & 0.175 \\
\hline Arterial Hypertension (\%) & 52.5 & 55.0 & 0.855 \\
\hline \multicolumn{4}{|l|}{ Ankle circumference $(\mathrm{cm})$} \\
\hline Left side & $22.4 \pm 2.1$ & $21.4 \pm 1.5$ & 0.070 \\
\hline Right side & $22.3 \pm 1.9$ & $21.2 \pm 1.4$ & 0.074 \\
\hline \multicolumn{4}{|l|}{ Calf circumference $(\mathrm{cm})$} \\
\hline Left side & $34.3 \pm 3.2$ & $33.6 \pm 2.6$ & 0.412 \\
\hline Right side & $34.7 \pm 3.0$ & $33.7 \pm 2.5$ & 0.193 \\
\hline Heart rate $(\mathrm{bpm})$ & $70.9 \pm 7.3$ & $68.2 \pm 6.6$ & 0.160 \\
\hline Systolic blood pressure $(\mathrm{mmHg})$ & $130 \pm 13$ & $126 \pm 9$ & 0.316 \\
\hline Diastolic blood pressure $(\mathrm{mmHg})$ & $75 \pm 1$ & $7 I \pm 2$ & 0.087 \\
\hline Body temperature $\left({ }^{\circ} \mathrm{C}\right)$ & $36.1 \pm 0.2$ & $36.1 \pm 0.2$ & 0.317 \\
\hline Perceived heaviness (scale) & $6.7 \pm 1.0$ & $6.9 \pm 1.0$ & 0.461 \\
\hline Comfort perception & $6.0 \pm 1.1$ & $4.5 \pm 0.8$ & $<0.001$ \\
\hline
\end{tabular}

\section{Statistical Analysis}

Continuous variables were reported as mean \pm standard deviation (SD) or median and interquartile range (IQR) based on their distribution. With respect to variables collected at hospitalization visit, comparison between groups A and B was assessed by unpaired $t$-test or Mann-Whitney test. Categorical variables were analyzed using the Chi-square test. Next, to capture the variability in repeated measures among the two study groups, we first measured the mean (and standard deviation) and the absolute difference between the final study visit and the baseline (hospitalization visit) for each variable. Next, we computed the coefficient of variation (CV, standard deviation/mean) which quantifies the mean variation of each variable during hospitalization. For skewed variables, $\mathrm{CV}$ was computed by replacing standard mean with geometric mean. Finally, we built multivariable linear regression analysis considering group A (versus group B as reference) as exposure and change in calf circumference, ankle circumference, perceived heaviness and comfort perception as endpoints. For the multivariable analysis, endpoints were modeled as an average of left and right legs where needed. Covariates were included in the multivariable linear analysis through a stepwise method with p-value threshold of $<0.05$. As subgroup analysis, we computed the differences of subjective parameters of heaviness and comfort perception specifically in groups with different degrees of leg elevation $\left(15^{\circ}\right.$ vs $\left.30^{\circ}\right)$. Data were analyzed using STATA 14 (StataCorp. College Station, TX, USA).

\section{Results}

The whole population consisted of 60 consecutive patients who met inclusion criteria of the study. Patients were distributed as follows: 40 undergoing leg elevation (group A) and 20 without leg elevation (group B). As shown in Table 1, the principal clinical and demographic features did not vary between group A and B (p-values $>0.05$ ), allowing an appropriate comparison in terms of the effect of elevation on the main study endpoints. Both groups were characterized by old age with mean age of 62.3 and 63.9 years in group A and B, respectively. Moreover, males were more frequently included than females in both groups. Frequency of comorbidities was not trivial, with arterial hypertension present in more than $50 \%$ of patients and diabetes in up to $45 \%$ of patients. Interestingly, comfort perception was higher in group A than in group B $(6.0 \pm 1.1 \mathrm{vs} 4.5 \pm 0.8, \mathrm{p}<0.001)$ still at baseline study visit.

Individual distribution of ankle and calf circumferences and comfort perception by type of in-hospital stay is represented in Figures 1 and 2. 


\section{A}

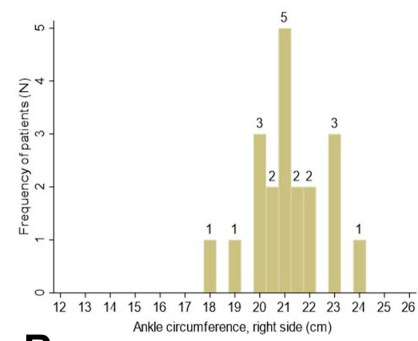

B
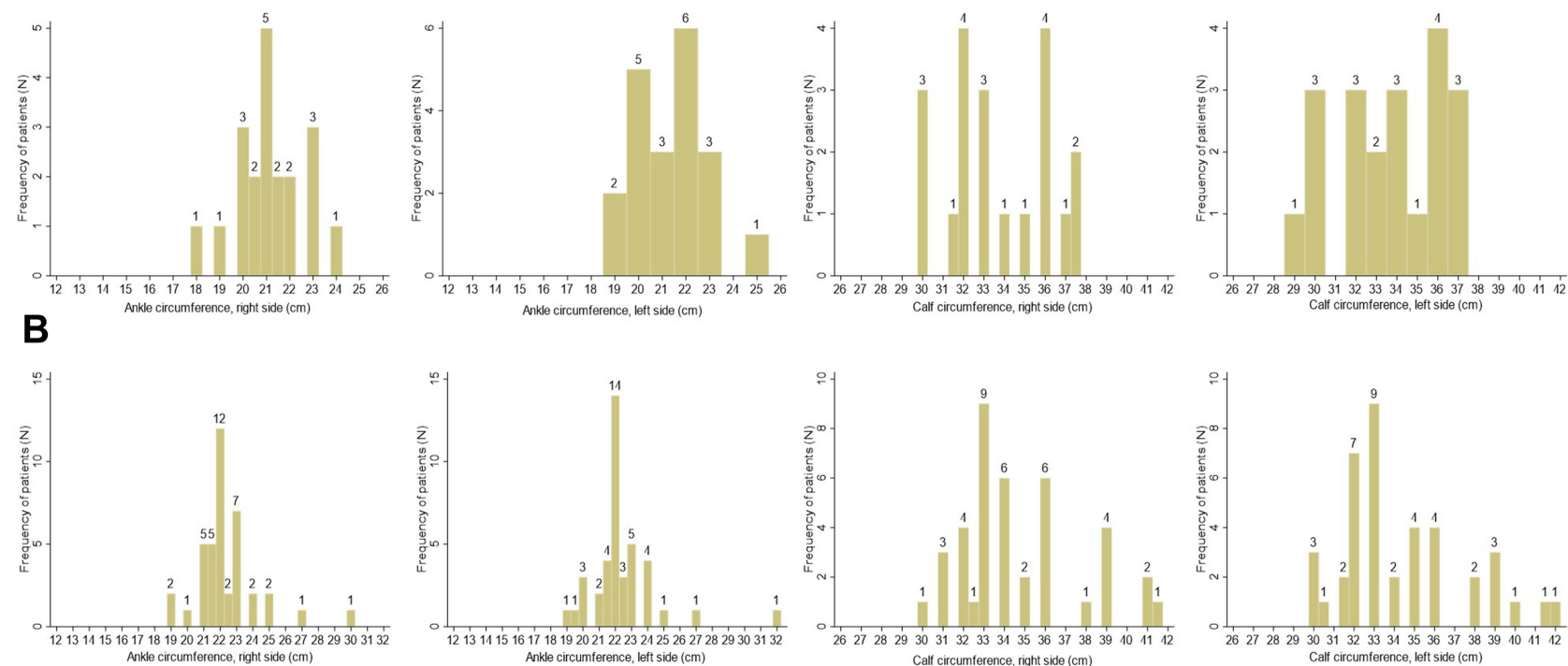

Figure I Distribution of ankle and calf distribution by type of in-hospital stay ((B) refers to patients with leg elevation and (A) refers to patients without leg elevation). Numbers over each column are the number of patients with the correspondent values reported on $\mathbf{x}$-axis.

A

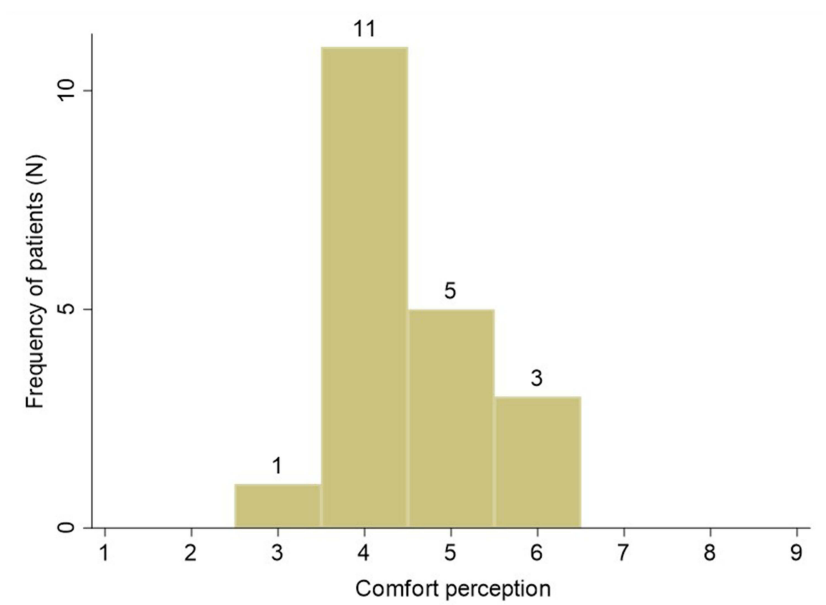

B

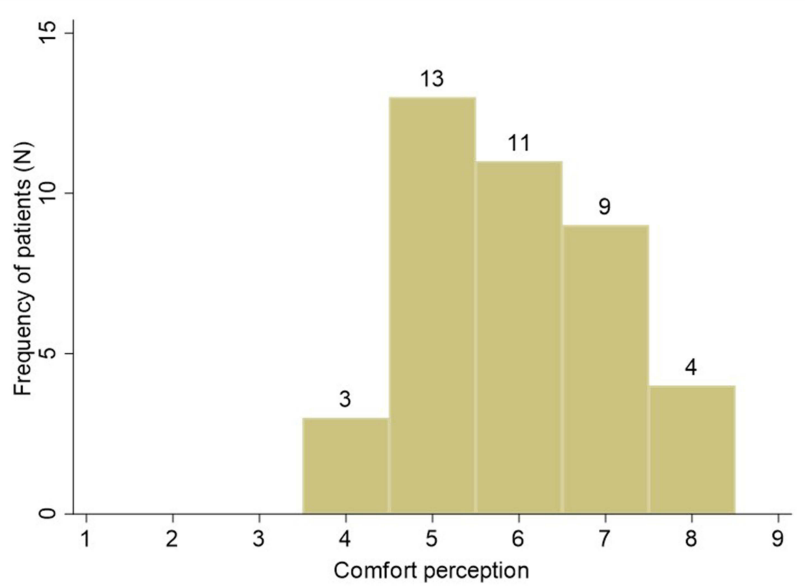

Figure 2 Distribution of comfort perception by type of in-hospital stay ((B) refers to patients with leg elevation and (A) refers to patients without leg elevation). Numbers over each column are the number of patients with the correspondent values reported on $\mathbf{x}$-axis.

When testing the changes between individual parameters measured day-by-day (Table 2), we found that delta in calf circumference (difference between end of study visit and baseline visit) were significantly decreased in group A vs group B $(\mathrm{p}<0.001$ for both sides). A similar finding was reported for the change in ankle circumference, which decreased by $2.7 \mathrm{~cm}$ and $2.8 \mathrm{~cm}$ in median in left and right legs as compared with group B (1 cm in median for both legs). In agreement with these changes, the coefficients of variation of calf and ankle circumference where significantly higher in group A than B, thus testifying to the effect of leg elevation on these changes. Both systolic and diastolic blood pressure decreased during in-hospital stay in patients of group A vs $B(p=0.028$ and $p=0.042$, respectively), whereas perceived heaviness decreased significantly in group A vs B ( -5 vs $1, \mathrm{p}<0.001)$. Conversely, comfort perception increased significantly during in-hospital stay in group A as compared with group $B(p=0.022)$. 
Table 2 Changes in Parameters Measured Day by Day During In-Hospital Stay

\begin{tabular}{|c|c|c|c|}
\hline & $\begin{array}{c}\text { Group A } \\
(\mathbf{N}=\mathbf{4 0})\end{array}$ & $\begin{array}{c}\text { Group B } \\
(\mathbf{N}=\mathbf{2 0})\end{array}$ & $\mathbf{P}$ \\
\hline \multicolumn{4}{|l|}{ Delta Calf circumference $(\mathrm{cm})$} \\
\hline Left side & $-2.5[-3--1.7]$ & $0.5[0.5-1.0]$ & $<0.001$ \\
\hline Right side & $-2.5[-3--1.5]$ & $0.5[0.3-1.5]$ & $<0.001$ \\
\hline $\mathrm{CV}$ calf circumference & $0.04 \pm 0.01$ & $0.01 \pm 0.00 \mathrm{I}$ & $<0.001$ \\
\hline \multicolumn{4}{|l|}{ Delta Ankle circumference $(\mathrm{cm})$} \\
\hline Left side & $-2.7[-3--2]$ & $\mathrm{I}[0.5-1.2]$ & $<0.001$ \\
\hline Right side & $-2.8[-3--2]$ & $\mathrm{I}[0.5-1.0]$ & $<0.001$ \\
\hline CV ankle circumference & $0.05 \pm 0.01$ & $0.01 \pm 0.001$ & $<0.001$ \\
\hline Delta Heart rate (bpm) & $3.0[-3.0-6.5]$ & $5.5[0.5-10.5]$ & 0.063 \\
\hline $\mathrm{CV}$ heart rate & $0.02 \pm 0.01$ & $0.03 \pm 0.01$ & 0.123 \\
\hline Delta Systolic blood pressure $(\mathrm{mmHg})$ & $-1.5[-7.5-2.5]$ & $5.0[0-5.0]$ & 0.028 \\
\hline CV systolic blood pressure & $0.01 \pm 0.001$ & $0.06 \pm 0.01$ & $<0.001$ \\
\hline Delta Diastolic blood pressure $(\mathrm{mmHg})$ & $-1.0[-5.5-3.0]$ & $4.0[0.3-4.0]$ & 0.042 \\
\hline CV diastolic blood pressure & $0.02 \pm 0.01$ & $0.05 \pm 0.01$ & 0.001 \\
\hline Delta Body temperature $\left({ }^{\circ} \mathrm{C}\right)$ & $0[-0.1-0.4]$ & $0.1[-0.2-0.1]$ & 0.675 \\
\hline $\mathrm{CV}$ body temperature & $0.02 \pm 0.01$ & $0.02 \pm 0.01$ & 0.365 \\
\hline Delta Perceived heaviness (scale) & $-5[-5--4]$ & $\mid[-|-|]$ & $<0.001$ \\
\hline CV perceived heaviness & $0.04 \pm 0.01$ & $0.008 \pm 0.001$ & $<0.001$ \\
\hline Delta Comfort perception (scale) & $2[0-4]$ & $0[-1-1]$ & $<0.001$ \\
\hline CV comfort perception & $0.02 \pm 0.001$ & $0.002 \pm 0.000 \mathrm{I}$ & 0.022 \\
\hline Delta hours of sleep $(\mathrm{hr})$ & $0[-1-1]$ & $0[0-1]$ & 0.562 \\
\hline CV hours of sleep & $0.004 \pm 0.0012$ & $0.002 \pm 0.0003$ & 0.712 \\
\hline
\end{tabular}

Abbreviation: $\mathrm{CV}$, coefficient of variation.

When assessing the multivariable linear regression analyses (Table 3), we found that leg elevation was a significant and independent correlate of change in calf circumference $(\beta=-3.21, \mathrm{p}<0.001)$, change in ankle circumference $(\beta=-3.64, \mathrm{p}<0.001)$, change in perceived heaviness $(\beta=-5.02, \mathrm{p}<0.001)$, and change in comfort perception $(\beta=1.63, \mathrm{p}=0.003)$. Regardless of leg elevation, we also found that arterial hypertension was a significant correlate of change in ankle circumference $(\mathrm{p}=0.040)$, whereas male gender was found as a possible determinant of change in perceived heaviness $(p=0.028)$.

As shown in Table 4, a higher degree of comfort perception was achieved in group A1 ( $15^{\circ}$ leg elevation) as compared with group A2 $\left(30^{\circ}\right.$ leg elevation) with an increase of 4 points and 1 point $(p<0.001)$, respectively. We did not find differences in delta of perceived heaviness between group A1 and A2 ( $\mathrm{p}=0.898)$.

Table 3 Multivariable Linear Regressions for the Correlates of Study Endpoints

\begin{tabular}{|c|c|c|c|}
\hline Change in Calf Circumference & B & $95 \% \mathrm{Cl}$ & $\mathbf{P}$ \\
\hline Elevation (group A vs B) & -3.21 & $-3.7|--2.7|$ & $<0.001$ \\
\hline Change in Ankle Circumference & B & $95 \% \mathrm{Cl}$ & $\boldsymbol{P}$ \\
\hline Elevation (group A vs B) & -3.64 & $-4.12--3.16$ & $<0.001$ \\
\hline Arterial hypertension & 0.49 & $0.02-0.95$ & 0.040 \\
\hline Change in Perceived Heaviness & B & $95 \% \mathrm{Cl}$ & $\boldsymbol{P}$ \\
\hline Elevation (group A vs B) & -5.02 & $-5.7 \mid--4.33$ & $<0.001$ \\
\hline Male gender & 1.01 & $0.12-1.89$ & 0.028 \\
\hline Change in Comfort Perception & B & $95 \% \mathrm{Cl}$ & $\boldsymbol{P}$ \\
\hline Elevation (group A vs B) & 1.63 & $0.57-2.69$ & 0.003 \\
\hline
\end{tabular}


Table 4 Changes in Subjective Parameters Measured Day by Day During In-Hospital Stay, by Subgroups of Degrees of Leg Elevation (Group AI Refers to $15^{\circ}$ Leg Elevation and Group A2 Refers to $30^{\circ}$ Leg Elevation)

\begin{tabular}{|c|c|c|c|}
\hline & $\begin{array}{c}\text { Group AI } \\
\mathbf{( N = 2 0 )}\end{array}$ & $\begin{array}{c}\text { Group A2 } \\
\mathbf{( N = 2 0 )}\end{array}$ & P \\
\hline Delta Perceived heaviness (scale) & $-5[-5--4]$ & $-5[-6--4]$ & 0.898 \\
CV perceived heaviness & $0.04 \pm 0.01$ & $0.04 \pm 0.01$ & 0.543 \\
Delta Comfort perception (scale) & $4[3-4]$ & $1[-1-2]$ & $<0.001$ \\
CV comfort perception & $0.03 \pm 0.003$ & $0.001 \pm 0.0001$ & 0.012 \\
\hline
\end{tabular}

\section{Discussion}

As humans are bipedal, long-limbed with elastic leg skin, the human venous system is more complicated than arteries and it strictly depends on peripheral muscle pumps that ensure the blood return against the force of gravity, and on a series of venous valves that prevent retrograde venous blood flow. During normal walking, three main muscular pumps (foot, calf, and thigh muscle pumps) force rhythmically venous blood proximally, and when muscle contraction ceases the volume of blood contained within the veins of the lower limbs increases. In sedentary settings and under several pathologic circumstances, persistent leg muscle pumps' dysfunction may lead to peripheral vein hypertension that, if not addressed, can lead to hemodynamic changes to the vein wall. ${ }^{2,3}$

Elevating lower limbs at or above heart level has been shown to improve venous circulation, even in healthy subjects, by means of decreasing venous pressure of the legs. ${ }^{8}$ The natural course of hospitalization may result in decreased patient physical activity with sedentary behavior, ${ }^{12}$ and this may lead to leg discomfort with temporary and mild venous signs and symptoms even in healthy people, due to induced venous muscle pumps' inactivity. ${ }^{8}$

Interestingly, the great majority of participants in this study were men and this can be easily explained since patients were referred to nephrology, cardiac and vascular surgery and, as we also selected patients without chronic venous disease (CVD), it is unlikely to find adult female patients without any signs of venous disease as CVD prevalence among females is up to $80 \%{ }^{7}$

In our study, patients with leg elevation (Group A) recorded a decrease in calf and ankle circumference and perceived less leg heaviness and even reported more comfortable legs compared to patients without leg elevation (Group B) that were affected by a certain increase in calf and ankle circumference during hospital stay, also reporting more exhausted legs.

Moreover, among patients of Group A, the bigger advantage in terms of comfort perception was recorded in patients with $15^{\circ}$ leg elevation, probably due to the minor stress to the articular leg joints, in respect to $30^{\circ}$ leg elevation, and this data need further research.

This study has some implications for nursing practice especially in the actual era of precision nursing, ${ }^{13}$ and in particular, even more specialized registered nurses, such as vascular nurses, ${ }^{14}$ need to evaluate patients' leg vascular status during hospital stay and should also adjust, during bed rest, leg elevation supporting healthy leg vein circulation. Therefore, it is conceivable that a slight leg elevation during hospital stay of at least $15^{\circ}$ may help venous return of hospitalized patients, improving leg discomfort due to scarce leg activity or inactivity and also prevent more serious problem of the vein systems of lower limbs that may be triggered by inactivity-induced venous stasis of lower limbs.

There are some limitations: while it seems clear that leg elevation induces beneficial effects in leg calf and ankle circumferences and perception of leg heaviness, the underpinning mechanisms still remain unclear. Moreover, the effects of repeated leg elevation (over several days to weeks) and other leg elevation protocols (changing the degree of elevation) have not been studied and, definitely, it would also be useful to validate these results in future studies which enroll a larger number of patients.

\section{Institutional Review Board Statement}

The study was conducted according to the guidelines of the Declaration of Helsinki, and approved by the Institutional Review Board of Interuniversity Center of Phlebolymphology (CIFL), International Research and Educational Program in Clinical and Experimental Biotechnology (Approval number: ER.ALL.2018.12.A). 


\section{Data Sharing Statement}

The data presented in this study are available on request from the corresponding author. The data are not publicly available because an electronic link to the data has not been created.

\section{Informed Consent Statement}

Informed consent was obtained from all subjects involved in the study.

\section{Author Contributions}

All authors made a significant contribution to the work reported, whether that is in the conception, study design, execution, acquisition of data, analysis and interpretation, or in all these areas; took part in drafting, revising or critically reviewing the article; gave final approval of the version to be published; have agreed on the journal to which the article has been submitted; and agree to be accountable for all aspects of the work.

\section{Funding}

This research received no external funding.

\section{Disclosure}

The authors declare no conflicts of interest.

\section{References}

1. Uhl JF, Gillot C. Anatomy of the veno-muscular pumps of the lower limb. Phlebology. 2015;30(3):180-193. doi:10.1177/0268355513517686

2. Alimi YS, Barthelemy P, Juhan C. Venous pump of the calf: a study of venous and muscular pressures. J Vasc Surg. 1994;20(5):728-735. doi:10.1016/S0741-5214(94)70160-1

3. Hague A, Pherwani A, Rajagopalan S. Role of compression therapy in pathophysiology of the venous system in lower limbs. Surgeon. 2017;15 (1):40-46. doi:10.1016/j.surge.2016.08.004

4. Corrigan HJ, Burns J, Huggett RJ, Mackintosh AF, Mary DASG. The direct effect of leg position on calf blood flow measured by venous occlusion plethysmography. Artery Res. 2008;2:44-48. doi:10.1016/j.artres.2007.11.001

5. Abu-Own A, Scurr JH, Coleridge Smith PD. Effect of leg elevation on the skin microcirculation in chronic venous insufficiency. $J$ Vasc Surg. 1994;20(5):705-710. doi:10.1016/S0741-5214(94)70157-1

6. González-Alonso J, Mortensen SP, Jeppesen TD, et al. Haemodynamic responses to exercise, ATP infusion and thigh compression in humans: insight into the role of muscle mechanisms on cardiovascular function. $J$ Physiol. 2008;586(9):2405-2417. doi:10.1113/jphysiol.2008.152058

7. Serra R, Grande R, Butrico L, Fugetto F, De Franciscis S. Epidemiology, diagnosis and treatment of chronic venous disease: a systematic review. Chirurgia. 2016;29(2):34-45.

8. Orhurhu V, Chu R, Xie K, et al. Management of lower extremity pain from chronic venous insufficiency: a comprehensive review. Cardiol Ther. 2021;10(1):111-140. doi:10.1007/s40119-021-00213-x

9. Li B, Wang G, Wang Y, Bai L. Effect of two limb positions on venous hemodynamics and hidden blood loss following total knee arthroplasty. J Knee Surg. 2017;30(1):70-74. doi:10.1055/s-0036-1579787

10. Koch Esteves N, Gibson OR, Khir AW, González-Alonso J. Regional thermal hyperemia in the human leg: evidence of the importance of thermosensitive mechanisms in the control of the peripheral circulation. Physiol Rep. 2021;9(15):e14953. doi:10.14814/phy2.14953

11. Consort Statement. Consolidated Standards of Reporting Trials (CONSORT) reporting guidelines. Available from: http://www.consort-statement. org/. Accessed October 14, 2021.

12. Kirk AG, Behm KJ, Kimmel LA, Ekegren CL. Levels of physical activity and sedentary behavior during and after hospitalization: a systematic review. Arch Phys Med Rehabil. 2021;102(7):1368-1378. doi:10.1016/j.apmr.2020.11.012

13. Ielapi N, Andreucci M, Licastro N, et al. Precision medicine and precision nursing: the era of biomarkers and precision health. Int $J$ Gen Med. 2020;13:1705-1711. doi:10.2147/IJGM.S285262

14. Ielapi N, Licastro N, Catana M, Bracale UM, Serra R. Vascular nursing and vascular surgery. Ann Vasc Surg. 2020;68:522-526. doi:10.1016/j. avsg.2020.05.038

Nursing: Research and Reviews

Dovepress

\section{Publish your work in this journal}

Nursing: Research and Reviews is an international, peer-reviewed, open access journal publishing original research, reports, reviews and commentaries on all aspects of nursing and patient care. These include patient education and counseling, ethics, management and organizational issues, diagnostics and prescribing, health outcomes, economics and resource management, improving patient safety in all settings. The manuscript management system is completely online and includes a very quick and fair peer-review system. Visit http://www.dovepress.com/ testimonials.php to read real quotes from published authors.

Submit your manuscript here: http://www.dovepress.com/nursing-research-and-reviews-journal 